\title{
Parametric exploration of intense positron beam-plasma interactions
}

\author{
B.E. BLUE, ${ }^{1}$ C.E. CLAYTON, ${ }^{1}$ C.L. O'CONNELL,${ }^{2}$ F.-J. DECKER,${ }^{2}$ M.J. HOGAN,${ }^{2}$ C. HUANG,${ }^{1}$ \\ R. IVERSON, ${ }^{2}$ C. JOSHI, ${ }^{1}$ T.C. KATSOULEAS,${ }^{3}$ K.A. MARSH, ${ }^{1}$ W.B. MORI, ${ }^{1}$ AND D. WALZ ${ }^{2}$ \\ ${ }^{1}$ Electrical Engineering Department, University of California, Los Angeles, California \\ ${ }^{2}$ Stanford Linear Accelerator Center, Stanford University, Stanford, California \\ ${ }^{3}$ Electrical Engineering Department, University of Southern California, Los Angeles, California \\ (ReCEIVED 20 February 2003; ACCEPTEd 1 May 2003)
}

\begin{abstract}
A systematic investigation of the longitudinal fields excited in a plasma by a short, dense beam of positrons is carried out using two-dimensional, cylindrical geometry, particle-in-cell code simulations. In particular, we examine the behavior of the accelerating and decelerating fields of the wakefield as a function of beam charge, radius, length, and plasma density. The parameters are chosen to be consistent with those employed in current and future experiments designed to elucidate the physics of positron beam-plasma interactions.
\end{abstract}

Keywords: Advanced accelerator; Beam-plasma, Plasma waves, Relativistic positron beams

\section{INTRODUCTION}

In a future plasma-based linear collider, both electrons and positrons must be accelerated and collided with center-ofmass energies and luminosities required of a machine at the so called energy frontier (Bagger \& Barish, 2002). One way to accelerate a positron beam at ultrahigh gradients to these energies is to create a wakefield in a plasma using a positron beam driver that can then accelerate a trailing (second) beam of positrons. In present proof-of-principle experiments (Joshi et al., 2002), no trailing beam is used. Rather, the drive beam itself is used to both excite and probe the plasma wake by analyzing the energy gain, from the accelerating field $\mathrm{E}^{+}$ and energy loss from the decelerating field $\mathrm{E}^{-}$, of different longitudinal slices of the beam.

In comparison to the body of literature on the interaction of intense electron beams with plasmas (Hammer \& Rostoker, 1970; Hairapetian et al., 1994; Esarey et al., 1995, 1996; Barov et al., 2000), there is relatively little known

Address correspondence and reprint requests to: Chan Joshi, 66-147G Engineering IV, University of California Los Angeles, 405 Hilgard Ave., Los Angeles, CA 90095, USA. E-mail: joshi@ee.ucla.edu about how intense positron beams interact with plasmas. This is mainly because such an interaction is inherently nonlinear and must therefore be explored through fully selfconsistent, particle-in-cell (PIC) code simulations. Some aspects of this problem have been explored recently (Lee et al., 2001) and tested experimentally ( $\mathrm{Ng}$ et al., 2002; Blue et al., 2003; Hogan et al., 2003) in connection with a plasma-based wakefield accelerator driven by an intense positron beam. In this article, we document systematically how the longitudinal electric fields induced in a plasma by a short, dense beam of positrons scales with beam charge, plasma density, beam radius, and beam bunch length. These scalings are important for understanding the outcome of recent experiments by Blue et al. (2003) on positron-induced plasma wakes in which a few picosecond long, $\sim 1 \mathrm{kA}$ peak current positron beam was used to excite this longitudinal field in a plasma, and for designing future experiments. Associated with this longitudinal field is the transverse field that varies both with radius and with the longitudinal position within the bunch, which will lead to the transverse evolution (focusing and defocusing) of the beam (Hogan et al., 2003) which will not be addressed in this article. 


\section{LINEAR THEORY}

Although the interaction of a dense $\left(n_{b}>n_{p}\right)$, short $\left(\sigma_{z} \sim\right.$ $\lambda_{p}$ ) positron beam with a plasma is inherently nonlinear, we can use the linear theory (Katsouleas et al., 1987) that predicts the plasma response to either a short electron or positron beam as a guide to interpret the PIC code results. Here $n_{b}, n_{p}, \sigma_{z}$, and $\lambda_{p}$ are the beam density, plasma density, beam longitudinal characteristic length, and the wavelength of the plasma response, respectively. The linear wakefield response of a plasma induced by a test charge moving at the speed of light $c$ can be calculated as follows.

Starting with Maxwell's equations, the equation of motion for the plasma electrons, and the continuity equation, we write an equation for the evolution of the perturbed plasma density as

$$
\frac{\partial^{2} n_{1}}{\partial t^{2}}+\omega_{p}^{2} n_{1}=\omega_{p}^{2} \frac{q}{e c} \delta(r) \delta\left(t-\frac{z}{c}\right) .
$$

Here $n_{1}, \omega_{p}, q$, and $e$ are the perturbed plasma density, the plasma frequency, the test particle charge, and the charge of an electron respectively. Equation (1) is solved by using the Laplace transform. This results in an equation that describes the response of the plasma to the test particle:

$$
n_{1}=\omega_{p} \frac{q}{e c} \delta(r) \sin \left[\omega_{p}\left(t-\frac{z}{c}\right)\right] U\left(t-\frac{z}{c}\right) .
$$

Here, $U(t)$ is the unit step function and we see that the plasma is perturbed sinusoidally at the plasma frequency after the beam passes. Next the wave equation for the electric field, which arises from the perturbed plasma density, can be written as

$$
\left(\nabla_{\perp}^{2}-k_{p}^{2}\right) \vec{E}_{1}=4 \pi q k_{p}^{2} \delta(r) U\left(t-\frac{z}{c}\right) \cos \left[\omega_{p}\left(t-\frac{z}{c}\right)\right] .
$$

The above equation is the Green's Function response of the modified Helmholtz equation in cylindrical geometry. Solving for the component of the electric field $E_{z}$ that propagates in the direction of propagation of the wave gives

$$
E_{Z}=-2 q k_{p}^{2} K_{0}\left(k_{p} r\right) U\left(t-\frac{z}{c}\right) \cos \left[\omega_{p}\left(t-\frac{z}{c}\right)\right] .
$$

Here $k_{p}$ and $K_{0}$ are the plasma wave number and modified Bessel function of the first kind. From the above equation, it can be seen that the test particle generates a wakefield in the plasma that oscillates at the plasma frequency. Because we are interested in the electric field generated by a finite length bunch that has an extended spatial distribution, we integrate the impulse response over a Gaussian distribution that is given by

$$
n_{b}(r, \zeta)=\frac{q}{(2 \pi)^{3 / 2} \sigma_{r}^{2} \sigma_{z}} \exp \left(-\frac{r^{2}}{\sigma_{r}^{2}}-\frac{\zeta}{2 \sigma_{z}^{2}}\right) .
$$

Here $\zeta=c t-z$ is the "beam coordinate," and $\sigma_{r}$ and $\sigma_{z}$ are the radial and longitudinal root mean square beam sizes, respectively. The above integration results in

$$
e E_{Z}=\sqrt{n_{p}} \frac{n_{b}}{n_{p}} \frac{|q|}{q} \frac{\sqrt{2 \pi} k_{p} \sigma_{z} e^{-k_{p}^{2} \sigma_{Z}^{2} / 2}}{1+\frac{1}{k_{p}^{2} \sigma_{r}^{2}}} \sin k_{p}(z-c t) \quad(\mathrm{eV} / \mathrm{cm}) .
$$

Several important scaling laws can be extrapolated from this equation. First, the wakefield excited by the bunch oscillates sinusoidally with a frequency determined by the plasma density and its phase velocity travels at the speed of light. Second, the accelerating gradient increases linearly with increasing charge. This is because the increased charge increases the strength of the beam space charge field, which in turn drives the plasma wave harder. Third, the fields excited by electrons and positron beams are equal in magnitude but opposite in phase. Finally, by examining the behavior of the first and second derivatives with respect to $z$ of Eq. (6), it can be seen that the field will be maximized for a value of $k_{p} \sigma_{z}=\sqrt{2}$.

\section{SCALING LAWS EXPLORED THROUGH PIC SIMULATIONS FOR A POSITRON BEAM}

Although the scaling laws inferred from Eq. (6) in the previous section are useful, the approximations made are often not entirely valid, as in the current experiments that are being carried out at the Stanford Linear Accelerator Center (SLAC). For instance, although the linear theory assumes that the density perturbation induced by the beam is typically small, the beam density can exceed the plasma density over most of the length of the bunch in current experiments, and therefore linear theory can break down. The dynamics of the plasma electrons that are being perturbed by the space charge field of the positron beam are extremely complicated. As plasma electrons from different radii outside of the bunch are pulled in by the space charge field of the positron beam they arrive at different times at different longitudinal locations within the beam. This mandates the use of PIC simulations to gain insight beyond that which was obtained from linear theory. The beam and plasma parameters used in the simulations will be the nominal parameters used in the SLAC E162 experiment (Joshi et al., 2002) which are listed in Table 1.

The PIC code OOPIC (Bruhwiler et al., 2001) was used to obtain the fields generated by the positron beam. Unless otherwise stated, the parametric study reported in this article was carried out by changing only one parameter in Table 1 while holding other parameters constant. OOPIC is a fully explicit, electromagnetic, two-dimensional, cylindrical geometry code. Typical simulation parameters were radial and longitudinal grid sizes $\Delta r=\Delta z=20 \mu \mathrm{m}$, time step $\Delta t=14 \mathrm{fs}, 20$ beam particles per cell, 12 plasma particles 
Table 1. Nominal beam parameters

\begin{tabular}{lc}
\hline \hline Nominal experimental parameter & Value \\
\hline Positron beam energy & $28.5 \mathrm{GeV}$ \\
Number of beam particles & $1.2 \times 10^{10} \mathrm{e}^{+}$ \\
Beam r.m.s. radius $\sigma_{r}$ & $40 \mu \mathrm{m}$ \\
Beam r.m.s. length $\sigma_{z}$ & $730 \mu \mathrm{m}$ \\
Plasma density $n_{p}$ & $1.8 \times 10^{14} \mathrm{e}^{-} / \mathrm{cm}^{3}$ \\
Peak beam density $n_{b 0}$ & $6.5 \times 10^{14} \mathrm{e}^{+} / \mathrm{cm}^{3}$ \\
\hline
\end{tabular}

per cell, and a simulation box with conducting boundaries $1 \mathrm{~mm}$ wide and $8 \mathrm{~mm}$ long. The singly ionized lithium ions were immobile and the initial plasma was cold. The wakefield was measured after the beam fully propagated into the plasma $\left(\sim 10 \sigma_{z}\right)$. At this point in the plasma, the wakefields are more or less fully excited and do not change shape or magnitude (in the beam frame) as the drive beam propagates further into the plasma, although parts of the beam itself can dramatically focus and defocus in the transverse direction in response to the wakefields (Hogan et al., 2003). In the longitudinal direction, there is energy gain or loss by positrons located at different phases of the beam, but at an initial energy of $28.5 \mathrm{GeV}$, the beam is "stiff" and the positrons do not physically move in the longitudinal direction. As the plasma electrons are attracted and pulled into the beam, the peak electron density within the bunch can exceed the beam density even though $n_{b}>n_{p}$, thus neutralizing the beam's space charge and setting up strong transverse and longitudinal wakefields. As mentioned in the introduction, we will discuss the scaling of the longitudinal field only in this article as a function of different beam and plasma parameters. As can be seen in Figure 1, for these beam parameters, approximately the front half of the beam sees a retarding or decelerating field $E^{-}$whereas the back half of the beam sees an accelerating field $E^{+}$. Consequently, the peak voltage transformer ratio $R=E^{+} / E^{-}$is approximately one as the front half of the beam losses energy to the wakefield and the back half of the beam extracts energy from the wakefield. The energy extraction efficiency can be quite high, as seen from the second accelerating peak that is left behind the drive bunch. This peak has half the amplitude of the first peak which in one dimension would mean that approximately $75 \%$ of the energy has been extracted from the wake by the accelerating particles. As mentioned earlier, wakefields produced after the beam has propagated $1 \mathrm{~cm}$ (dotted line) and $14 \mathrm{~cm}$ (solid line) are almost identical.

Figure 2 shows how the peak decelerating field $E^{-}$(triangles) varies with charge of the positron beam as the number of positrons, charge $/ q$, is varied between $1 \times 10^{9}$ and $3 \times$ $10^{10}$. The field increases monotonically with increasing charge. This is close to the prediction of the linear theory where the field strength would increase linearly with charge. In addition, the temporal location of the peak field is shown (squares). The location moves towards the head of the bunch

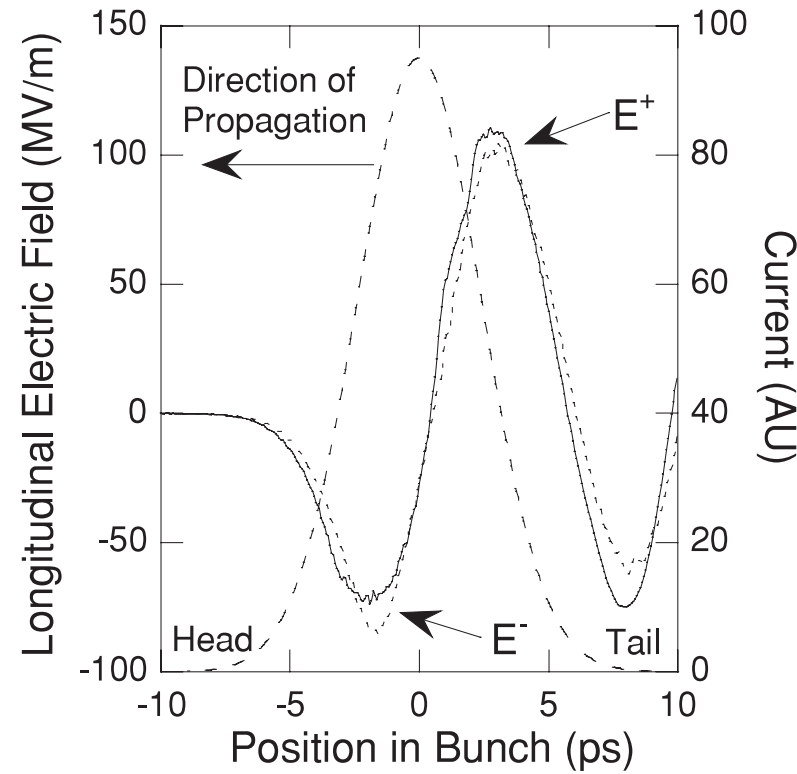

Fig. 1. Longitudinal wakefield excited on axis by a positron beam (dashed line) propagating from right to left after $1 \mathrm{~cm}$ (dotted line) and $14 \mathrm{~cm}$ (solid line) in a plasma. The notations used in Figures 2-10 are depicted in this figure.

again monotonically with increasing charge. As the charge is increased, the ratio of beam density to plasma density is increased. The subsequent increase in the space-charge field of the positron beam causes the plasma electrons to be pulled into the bunch sooner, thus leading to the location of the peak decelerating field moving forward.

Figure 3 shows how the peak accelerating field $E^{+}$(triangles) varies with charge. As before, the number of positrons is varied between $1 \times 10^{9}$ and $3 \times 10^{10}$. Consistent with

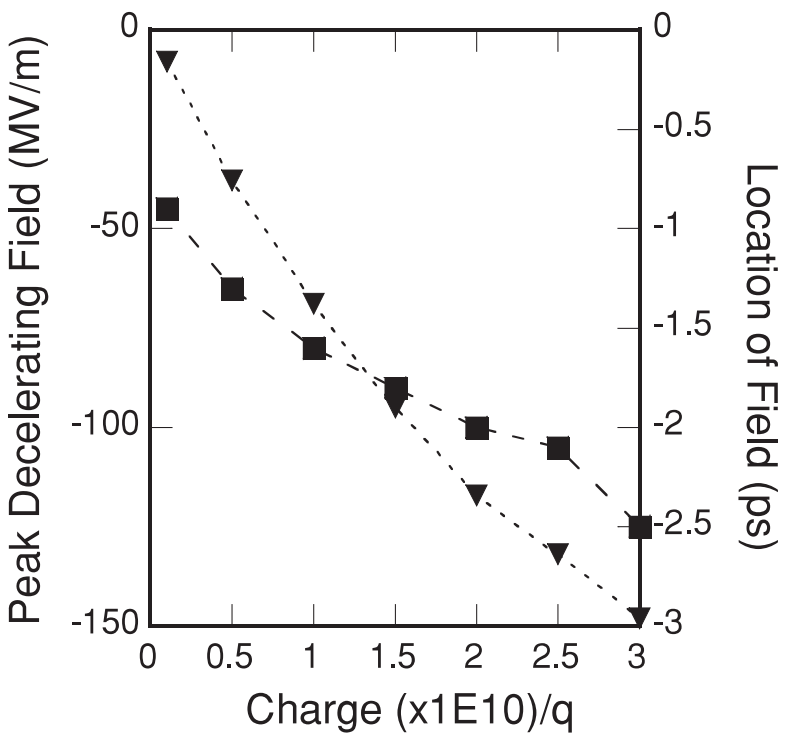

Fig. 2. Peak decelerating field (triangles) and its temporal location in the bunch (squares) versus charge. 


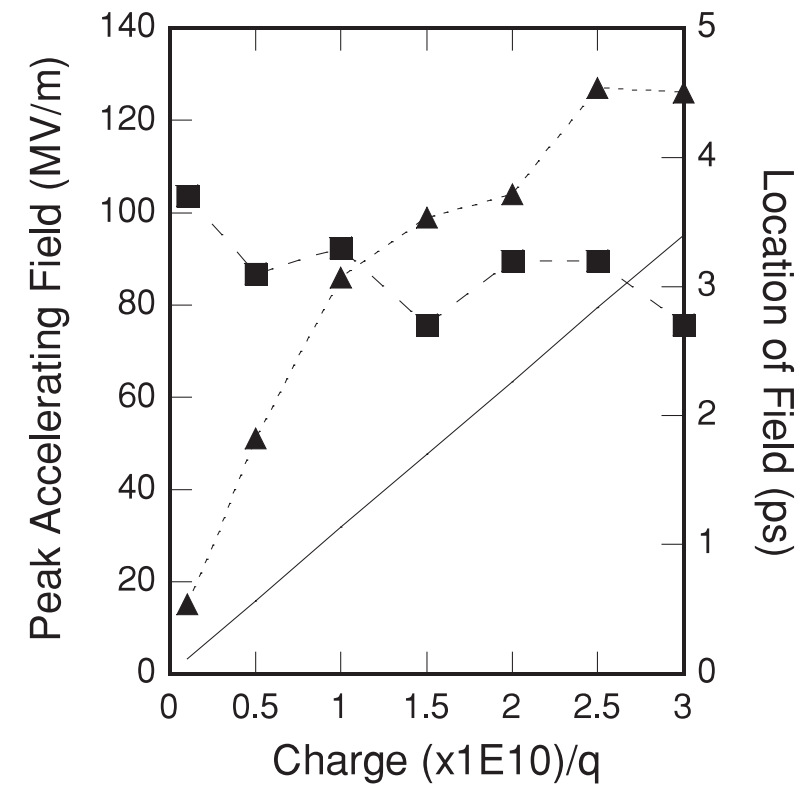

Fig. 3. Peak accelerating field (triangles) and its temporal location in the bunch (squares) versus charge. The linear theory prediction for the peak accelerating field is shown as a solid line.

the decelerating field, the peak accelerating field is also seen to increase with increasing charge; however, the field strength increases linearly up to a charge $/ q$ of $1 \times 10^{10}$, but then it begins to saturate with increasing charge. Whereas linear theory (solid line) predicted a linear growth of the field strength, it did not predict the saturation predicted by the PIC code. This saturation is due to the phase mixing of the plasma electrons pulled in by the beam as they oscillate about the beam axis. Furthermore, linear theory underestimates the field strength because of the greater role of the radial fields of the beam. The temporal location of the peak accelerating field (squares) changes less than \pm 0.5 ps with increasing charge, in contrast to $E^{-}$, which moves forward in the beam as mentioned earlier. It is important to note that for an electron beam driver, as one transitions from the linear $\left(n_{b} \ll n_{p}\right)$ to the highly nonlinear $\left(n_{b} \gg n_{p}\right)$ regime, the accelerating field rapidly increases as the beam density (or the drive beam charge for a given spot size) is increased, because the plasma electrons that are blown out by the beam head return within a narrow range of arrival times, which forms a density (and therefore electric field) spike on axis (Joshi et al., 2002). In contrast, there is a broad range of arrival times of the plasma electrons pulled in by the positron beam, and therefore, there is no sharp spike in the peak accelerating field $E^{+}$as seen in Figure 1.

Figure 4 shows how the peak decelerating field $E^{-}$(triangles) varies with increasing plasma density. The strength of the field increases rapidly up until a peak value of -86 $\mathrm{MV} / \mathrm{m}$ at a density of $1.1 \times 10^{14} \mathrm{~cm}^{-3}$. This density satisfies the $k_{p} \sigma_{z}=\sqrt{2}$ condition from linear theory for peak field. As the density is further increased, the strength of the field slowly decreases as predicted from Eq. (6). The temporal

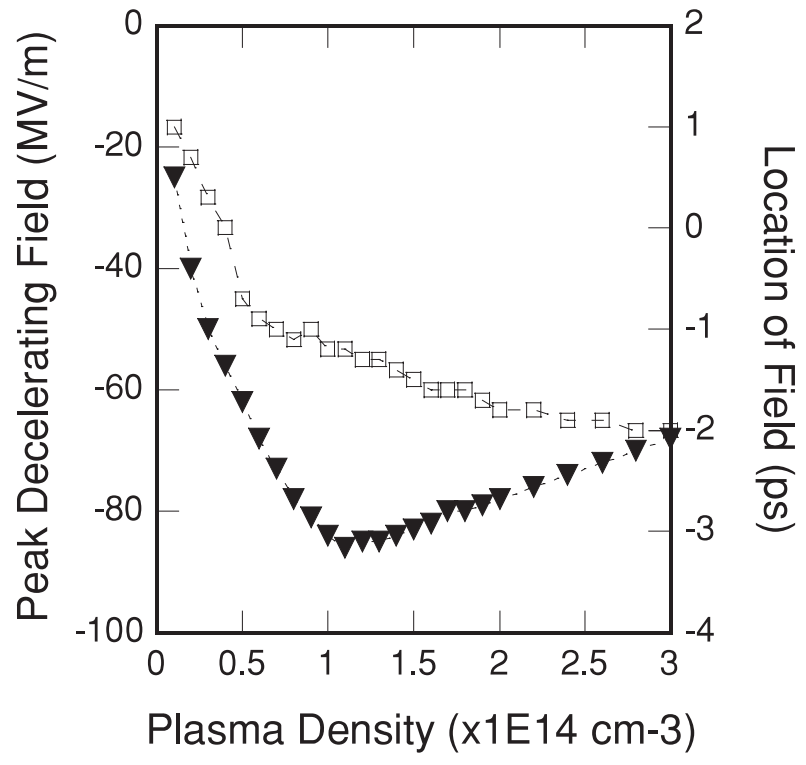

Fig. 4. Peak decelerating field (triangles) and its temporal location in the bunch (squares) versus plasma density.

location of the field (squares) moves from the back of the bunch towards the head of the bunch as the density in increased. This latter behavior is expected because the plasma wavelength is decreasing as the plasma density is increasing.

Figure 5 depicts the peak accelerating field $E^{+}$(triangles) as a function of plasma density. The strength of the field rapidly increases up to a peak value of $103 \mathrm{MV} / \mathrm{m}$ at a density of $9 \times 10^{13} \mathrm{~cm}^{-3}$. This value of density is close to that which is predicted by linear theory (solid line). Al-

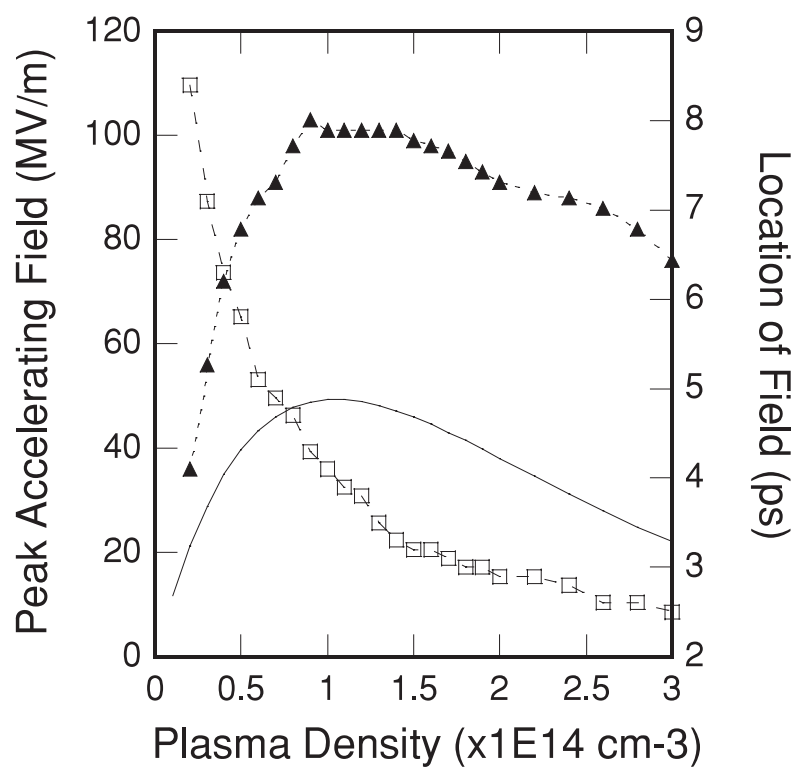

Fig. 5. Peak accelerating field (triangles) and its temporal location in the bunch (squares) versus plasma density. The linear theory prediction for the peak accelerating field is shown as a solid line. 
though the functional dependence of the peak accelerating field on plasma density predicted by linear theory is similar to the results obtained using PIC simulations, it underestimates the strength of the field. As the density is further increased beyond the optimal value, the peak field decreases as predicted by Eq. (6). The temporal location (squares) of this peak field moves from the back of the pulse toward the head of the bunch as the density is increased, as the plasma wavelength is decreasing.

Figure 6 shows the width of the accelerating bucket (circles) versus plasma density. The accelerating bucket is defined as the length of the field where the sign of the field is positive. According to linear theory developed in the previous section, the wake has a wavelength of $\lambda_{p}=c / f_{p}$. Because the plasma wavelength scales as $1 / \sqrt{n}$, it will decrease with increasing plasma density. The accelerating bucket would then have a width given by $\lambda_{p} / 2$. The half wavelength is shown as the solid line in the plot. The simulations show that at low densities, the wavelength is close to the value that is predicted by linear theory; however, at higher plasma densities the accelerating buckets are longer than that predicted by linear theory.

Figure 7 shows how the number of accelerated particles increases as plasma density is increased. As was shown in Figure 5, the temporal location of the peak field moves from the tail towards the centroid of the bunch as the plasma density is increased. Therefore, because the pulse shape is Gaussian, more and more particles will be accelerated (squares). The number of accelerated particles is calculated by taking the number of particles in a 1-ps bin centered at the temporal location of the peak field. For instance, five times more particles are accelerated at a density of $2 \times 10^{14} \mathrm{~cm}^{-3}$ than at $9 \times 10^{13} \mathrm{~cm}^{-3}$ with only

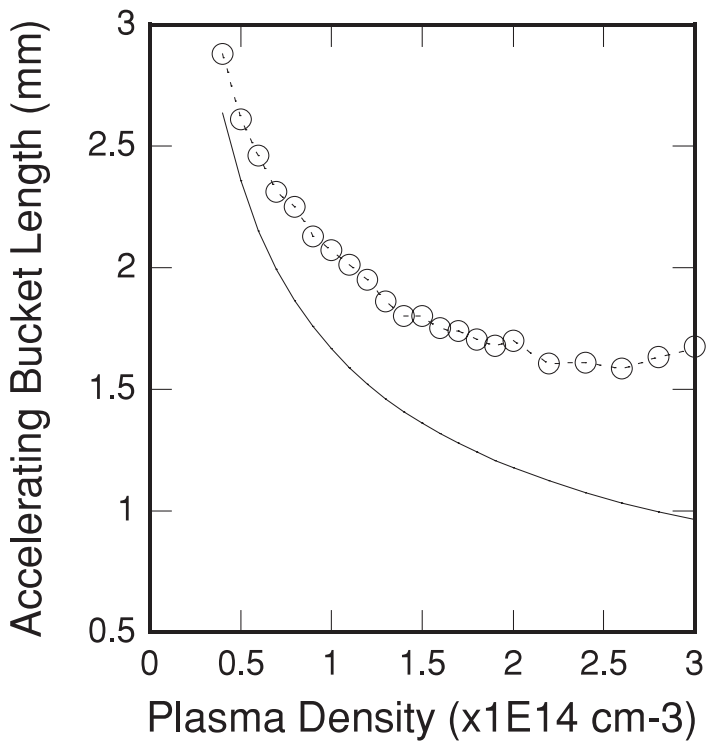

Fig. 6. Accelerating bucket width (circles) versus plasma density. The linear theory prediction of half a plasma wavelength is shown as a solid line.

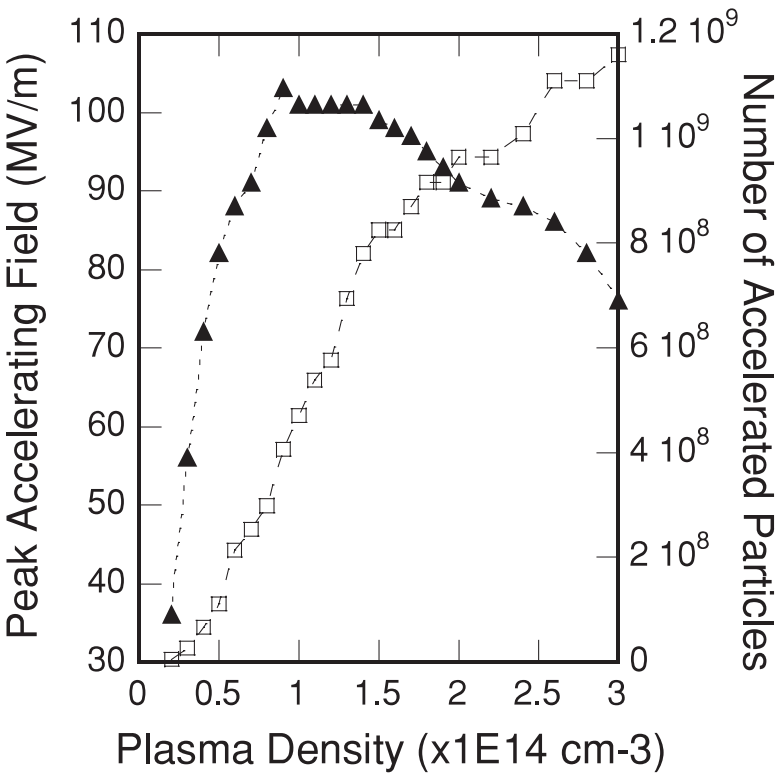

Fig. 7. Peak accelerating field (triangles) and accelerated charge in the 1 -ps peak field bin (squares) versus plasma density.

a $10 \%$ reduction in the peak field. At the optimum density of $9 \times 10^{13} \mathrm{~cm}^{-3}$, almost $3 \%$ of the total number of particles are within a picosecond wide slice situated about the peak accelerated field. In experiments in which a single bunch is used to both excite and witness the plasma wakefield, this number of accelerated particles is important. This is due to the need to have sufficient signal strength (accelerated positrons) in order to overcome the noise in the diagnostics (usually photoelectrons on a streak camera; Blue et al., 2003).

Figure 8 shows how the peak decelerating field $E^{-}$(triangles) changes as the radius of the beam is varied. As seen in the figure, the field decreases as the radius is increased. For instance, when the radius is increased from $40 \mu \mathrm{m}$ (the typical value in the experiment) by a factor of 4 to $160 \mu \mathrm{m}$, the field is decreased by a factor of 2 . According to linear theory, as long as the bunch radius is much less than the collisionless skin depth, the decelerating field within the bunch should be independent of the beam radius. Because the radii used in the simulations are much less than $c / \omega_{p}=$ $400 \mu \mathrm{m}$, this decrease is due to the changes in the beam charge density, which, in turn, affects the radial distribution of the plasma electrons pulled in by the beam and therefore the work the beam does on the these electrons. Also shown in Figure 8 is how the peak accelerating field (squares) changes as the bunch radius is varied. As expected from the behavior of the decelerating field, as the radius is increased, the accelerating field strength is also decreased. As discussed earlier, the change in the field magnitude is due to both a change in the beam charge density and to the dynamics of the plasma electrons being pulled in from different radii. 


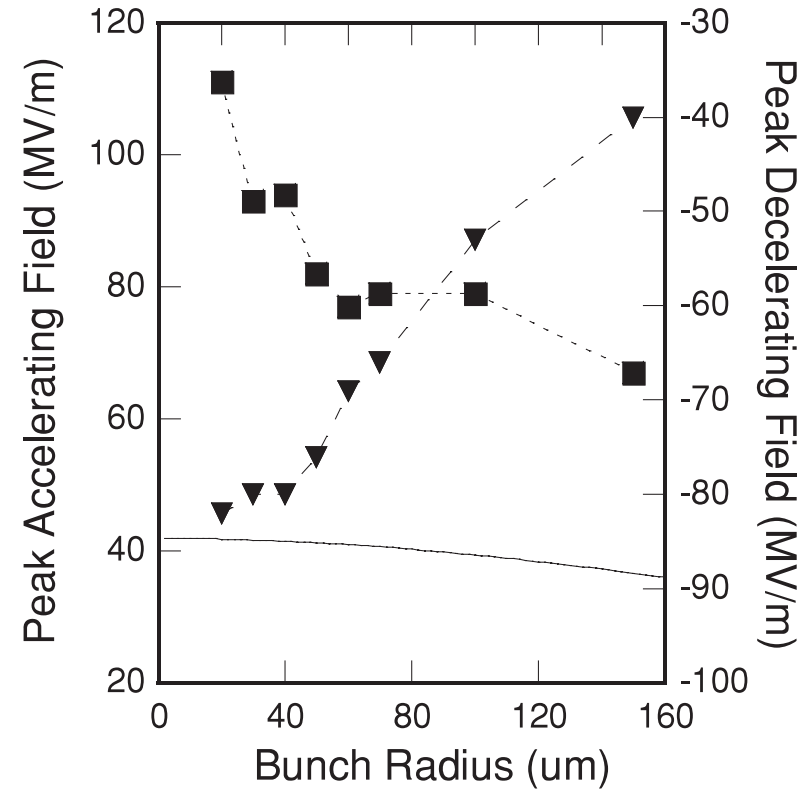

Fig. 8. Peak accelerating (squares) and decelerating (triangles) fields versus bunch radius. The linear theory prediction for the peak accelerating field is shown as a solid line.

Figure 9 shows the dependence of the peak decelerating field (triangles) on the bunch length. The field strength increases as the bunch length is decreased. According to the linear theory discussed in the preceding section, the field strength increases as $1 / \sigma_{z}^{2}$. The data was fitted with a $1 / \sigma_{z}^{2}$

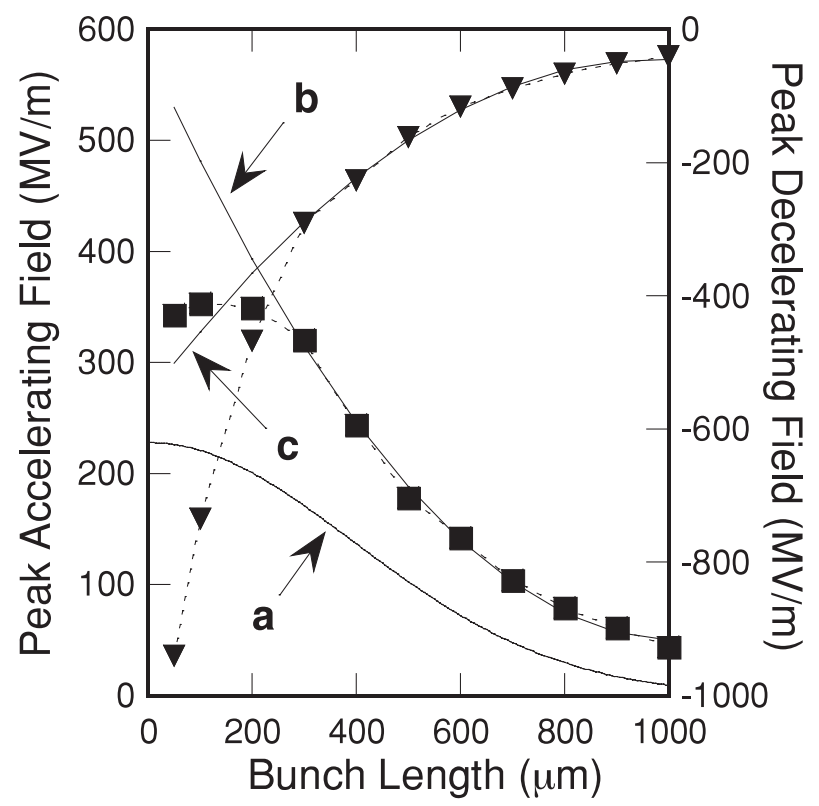

Fig. 9. Peak accelerating (squares) and decelerating (triangles) fields versus bunch length. The linear theory prediction for the peak accelerating field (a) is shown as a solid line. The inverse quadratic dependence for the peak accelerating field (b) and the peak decelerating field (c) are shown as solid lines. dependence by taking the field at $\sigma_{z}=1000 \mu \mathrm{m}$ point as a reference point (solid line c). As inferred from the plot, the field strength does have an inverse quadratic dependence up to a bunch length of $300 \mu \mathrm{m}$. For bunches shorter than this, the peak decelerating field seen in the simulations increases much more rapidly than what was predicted by linear theory.

Figure 9 also shows the dependence of the peak accelerating field (squares) on the bunch length. The field strength increases as the bunch length is decreased. According to the linear theory (solid line a) developed in the preceding section, the field strength $E^{+}$seen in the simulations also increases as $1 / \sigma_{z}^{2}$ (solid line b), but its magnitude is larger than that predicted by the linear theory. Note that for bunch lengths shorter than $300 \mu \mathrm{m}$, while the decelerating field actually increases faster than $1 / \sigma_{z}^{2}$, the accelerating field saturates.

\section{OPTIMIZED PLASMA WAKEFIELD ACCELERATION OF POSITRONS}

In the preceding section, scaling laws were developed in which a single beam or plasma parameter was varied while the others were held constant. Two important results were that the accelerating field increased quadratically as the bunch length was decreased (Fig. 9) and that each bunch length had a corresponding plasma density that maximized the accelerating field (Fig. 5). Therefore, we have tried a twoparameter optimization of the peak accelerating field by simultaneously reducing the bunch length and increasing the plasma density using the linear theory relationship $k_{p} \sigma_{z}=$ $\sqrt{2}$. The results for the peak accelerating field (triangles) are shown in Figure 10a. The solid curve shows the optimized plasma density from the linear theory. Now for $\sigma_{z}=50-\mu \mathrm{m}-$ long bunches, peak accelerating fields of $2.7 \mathrm{GeV} / \mathrm{m}$ can be obtained from a $40-\mu \mathrm{m}$ round beam with $1.2 \times 10^{10}$ positrons in a $2 \times 10^{16} \mathrm{~cm}^{-3}$ plasma. The longitudinal wakefield at this density is relatively sinusoidal as opposed to the electron beam case, where it is highly nonlinear (Lee et al., 2000). In Figure 10b, we plot the peak decelerating field (circles) that accompanied the accelerating field as well as the transformer ratio $R=E^{+} / E^{-}$for different values of bunch lengths used in Figure 10a. As the bunch length is shortened, the transformer ratio increases from 1 (energy loss equals energy gain) to a value of 1.6 with a $50-\mu \mathrm{m}$-long bunch. This means as bunch length is decreased, one benefits from both an increased acceleration field as well as a higher energy gain to energy loss ratio.

Further increase in the accelerating field can result from propagating the positron beam in a hollow channel as has been shown in other PIC simulations (Lee et al., 2001), and higher transformer ratios could result from using tailored shapes for the drive beam instead of a symmetric Gaussian pulse. These issues are currently being explored. The stability of the drive beam against transverse beam break-up instabilities, such as the transverse two-stream instability, is also a critical issue that needs further work. 

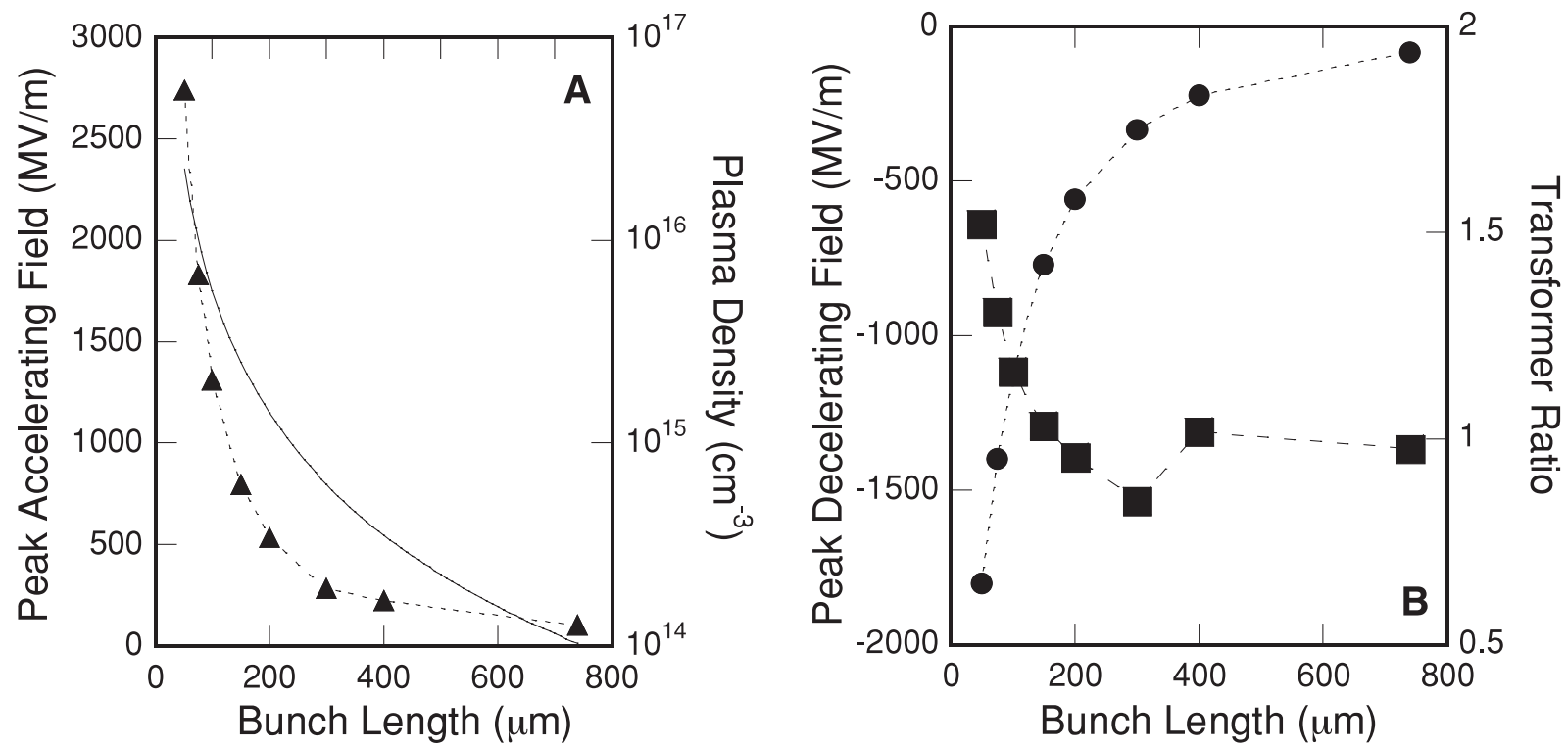

Fig. 10. Peak accelerating field (triangles), decelerating field (circles), and transformer ratio (squares) versus bunch length. The matched plasma density for the peak accelerating field is shown as a solid line.

\section{SUMMARY}

In this article, we have examined through PIC simulations the scaling of the longitudinal accelerating and decelerating fields of the wake produced by a positron beam in a plasma as a function of different beam and plasma parameters. Wherever possible, we have tried to compare the results with the predictions of linear theory which hold true for wakes excited by either an electron or a positron beam. However, we find that for the range of the beam and plasma parameters used the in current experiments, the positron beam-plasma interaction is inherently nonlinear, and only fully selfconsistent PIC code modeling can give the actual fields. We have shown here that once established, these fields do not significantly evolve and therefore the actual energy gain or loss seen by a particular beam slice is simply given by the local electric field times the distance traveled in the plasma. The study documented here has led to identification of the optimum parameter space for conclusively measuring the acceleration of positrons by a plasma wakefield for the first time by Blue et al. (2003).

\section{ACKNOWLEDGMENTS}

This work was support by the United States Department of Energy and the National Science Foundation. We thank David Bruhwiler of Tech-X Corporation for the use of the OOPIC Pro PIC code and Drs. P. Muggli and R. Siemann for their interest in this work.

\section{REFERENCES}

Bagger, J. \& Barish, B. (2002). The High-Energy Physics Advisory Panel long range plan for U.S. high-energy physics in the 21st century. http://doe-hep.hep.net/lrp_panel/hepap_lrp.pdf.
Barov, N., Rosenzweig, J.B., Conde, M.E., Gai, W. \& Power, J.G. (2000). Observation of plasma wakefield acceleration in the underdense regime. Phys. Rev. ST Accel. Beams 3, 011301:1-10.

Blue, B.E., Clayton, C.E., O’Connell, C.L., Decker, F.-J., Hogan, M.J., Huang, C., Iverson, R., Joshi, C., Katsouleas, T.C., Lu, W., Marsh, K.A., Muggli, P., Siemann, R.H. \& WALSH, D. (2003). Plasma-wakefield acceleration of an intense positron beam. Phys. Rev. Lett. 9, 4801.

Bruhwiler, D.L., Giacone, R.E., Cary, J.R., Verboncoeur, J.P., Mardahl, P., Esarey, E., Leemans, W.P. \& Shadwick, B.A. (2001). Particle-in-cell simulations of plasma accelerators and electron-neutral collisions. Phys. Rev. ST Accel. Beams 4, 101302:1-13.

Esarey, E., Sprangle, P., Krall, J. \& Ting, A. (1996). Overview of plasma-based accelerator concepts. IEEE Trans. Plasma Sci. 24, 252-288.

Hairapetian, G., Davis, P., Clayton, C.E., Joshi, C., HartMAN, S.C. \& Pellegrini, C. (1994). Experimental demonstration of dynamic focusing of a relativistic electron bunch by an overdense plasma lens. Phys. Rev. Lett. 72, 2403-2406.

Hairapetian, G., Davis, P., Clayton, C.E., Joshi, C., PelleGRINI, C. \& KATSOUlEAS, T. (1995). Transverse dynamics of a short, relativistic electron bunch in a plasma lens. Phys. Plasmas 2, 2555-2561.

Hammer, D. A. \& Rostoker, N. (1970). Propagation of high current relativistic electron beams. Phys. Fluids 13, 1831-1850.

Hogan, M.J., Clayton, C.E., Huang, C., Muggli, P., Wang, S., Blue, B.E., Walz, D., Marsh, K.A., O'Connell, C.L., Lee, S., Iverson, R., Decker, F.-J., Raimondi, P., Mori, W., Katsouleas, T.C., Joshi, C. \& Siemann, R.H. (2003). Ultrarelativistic-positron-beam transport through meter-scale plasmas. Phys. Rev. Lett. 90, 5002.

Joshi, C., Blue, B., Clayton, C. E., Dodd, E., Huang, C., Marsh, K. A., Mori, W.B. Wang, S., Hogan, M.J., O’Connell, C., Siemann, R., Walz, D., Muggli, P., Kat- 
SOUleAs, T. \& LEE S. (2002). High energy density plasma science with an ultrarelativistic electron beam. Phys. Plasmas 9, 1845-1855.

Katsouleas, T., Wilks, S., Chen, P., Dawson, J.M. \& Su, J.J. (1987). Beam loading in plasma accelerators. Part. Accel. 22, 81-99.

Lee, S., Katsouleas, T., Hemker, R.G., Dodd, E.S. \& Mori, W.B. (2001). Plasma-wakefield acceleration of a positron beam. Phys. Rev. E 64, 045501:1-4.
Lee, S., Katsouleas, T., Hemker, R.G. \& Mori, W.B. (2000). Simulations of a meter-long plasma wakefield accelerator. Phys. Rev. E. 61, 7014-7021.

Ng, J.S.T., Chen, P., Baldis, H., Bolton, P., Cline, D., Craddock, W., Crawford, C., Decker, F.J., Field, C., Fukui, Y., Kumar, V., Iverson, R., King, F., Kirby, R.E., Nakajima, K., Noble, R., Ogata, A., Raimondi, P., Walz, D. \& WeideMANN, A.W. (2001). Observation of plasma focusing of a 28.5 GeV positron beam. Phys. Rev. Lett. 87, 244801:1-4. 